\title{
Chemospecific reaction of activated acetylenic compounds with triphenylphosphine in the presence of a system containing two functional groups
}

\author{
Mohammad Reza Islami, ${ }^{a^{*}}$ Mohammad Ali Amrollahi, ${ }^{\text {and Maryam Iranmanesh }}{ }^{\mathrm{b}}$ \\ ${ }^{a}$ Department of Chemistry, Shahid Bahonar University of Kerman, Kerman, 76169 Iran \\ ${ }^{b}$ Department of Chemistry, School of Science, Yazd University, Yazd, Iran \\ E-mail:mrislami@mail.uk.ac.ir
}

\begin{abstract}
A mild and efficient chemospecific method has been developed for the preparation of organophosphorus compounds, in good yields, through the reaction of 2-bromoacetamide or ethyl phenylcarbamate with $\mathrm{PPh}_{3}$ in the presence of activated acetylenic esters.
\end{abstract}

Keywords: Chemospecific reaction, organophosphorus, 2-bromoacetamide, acetylenic esters

\section{Introduction}

Organophosphorus compounds have emerged as important reagents and intermediates in organic synthesis. ${ }^{1}$ An important group of this class is phosphorus ylides, which have been used in many reactions and synthesis of organic compounds. ${ }^{2-8}$ The prominent role of these compounds is to convert the carbonyl groups to carbon-carbon double bonds. ${ }^{9,10}$ From the large number of methods available for the synthesis of phosphorus ylides, the most important involve the reaction of a phosphonium salt with a base. ${ }^{11,12}$ In recent years a method has been developed for the preparation of this family by using a novel approach employing vinyl phosphonium salts. ${ }^{13-16}$ Although this method is successful for the preparation of phosphorus ylides and 1,4-di-ionic organophosphorus compounds ${ }^{17}$ from one $\mathrm{CH}$-acid or $\mathrm{NH}_{2}$ groups ${ }^{18,19}$ there are few reports on the chemoselectivity and chemospecific synthesis of these compounds. ${ }^{20}$ Hence, a convenient chemospecific synthesis of phosphorus compounds in general, and functionalized phosphorus ylides in particular, continues to be a challenging task. We have found that 2-bromoacetamide reacts with triphenylphosphine in the presence of acetylenic esters to produce stable $\alpha$-amido phosphorus ylides in a chemospecific manner, with the $\mathrm{CH}_{2} \mathrm{Br}$ moiety remaining intact. The $\alpha$ amido phosphorus ylides have also been prepared by reaction of triphenylphosphine with ethyl phenylcarbamate as an amido group, in the presence of dialkyl acetylenedicarboxylates. 


\section{Results and Discussion}

Both neutral and anionic phosphorus compounds are good nucleophiles ${ }^{21}$ toward alkyl halides and $\alpha$-halocarbonyl compounds. The reaction of some $\alpha$-halocarbonyl compounds such as $\alpha$ bromoketones with phosphines and phosphate can take an alternative course in which phosphorus attacks halogen, with the formation, respectively, of an enolate or an enol phosphate. $^{22,23}$ Also, the nucleophilic substitution reactions by a phosphorus atom can occur at an $\mathrm{sp}^{3}$ - carbon bearing halogen, and are most valuable for synthesis. ${ }^{21}$ When a synthesis is being undertaken with several sensitive functional groups present in the molecule, or with several sensitive molecules, milder reagents and reaction conditions may be necessary. As a result, many alternative methods for effecting syntheses of phosphorus ylides from carbonyl compounds have been developed. It is worth mentioning that although the reaction of $\mathrm{PPh}_{3}$ with compounds containing halogen groups, such as $\alpha$-bromoketones, ${ }^{22}$ carbon tetrachloride, and hexachloroacetone, ${ }^{23-26}$ has been reported in the literature, we have not observed products derived from nucleophilic attack of the $\mathrm{PPh}_{3}$ at the $\mathrm{CH}_{2} \mathrm{Br}$ group in 2-bromoacetamide. Apparently, under the present reaction conditions the phosphonium ion $\mathbf{3}$, which is conjugated with an $\alpha, \beta$-unsaturated ester, is formed faster than salt $\mathbf{4}$, and the compound $\mathbf{5}$ is formed in a chemospecific manner (Scheme 1).
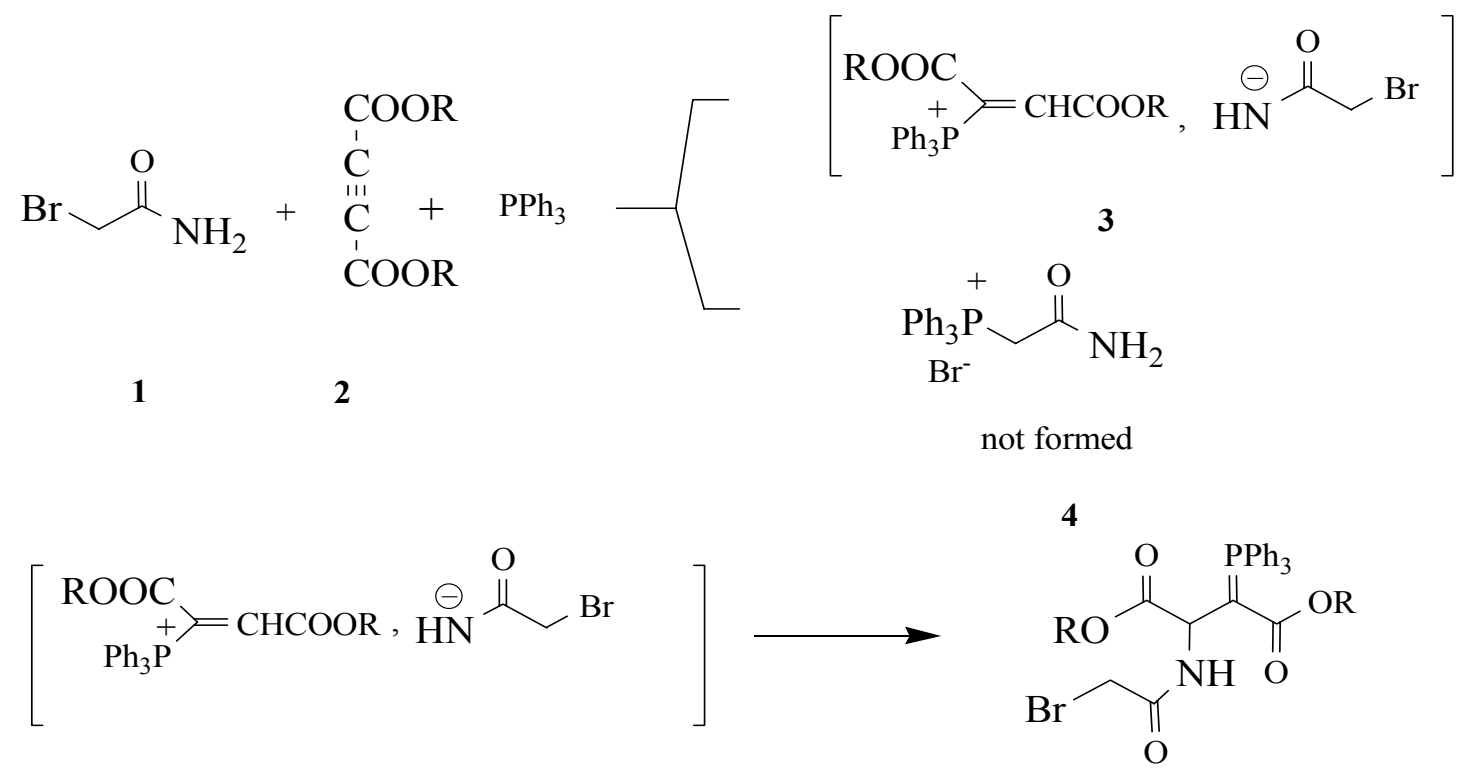

5a $\mathrm{R}=\mathrm{Me}$

$5 \mathbf{b} \quad \mathrm{R}=\mathrm{Et}$

\section{Scheme 1}


In order to investigate chemospecific reactions of carbamate for the preparation of phosphorus ylides, the reaction of ethyl phenylcarbamate with acetylenic esters was carried out in the presence of triphenylphosphine.
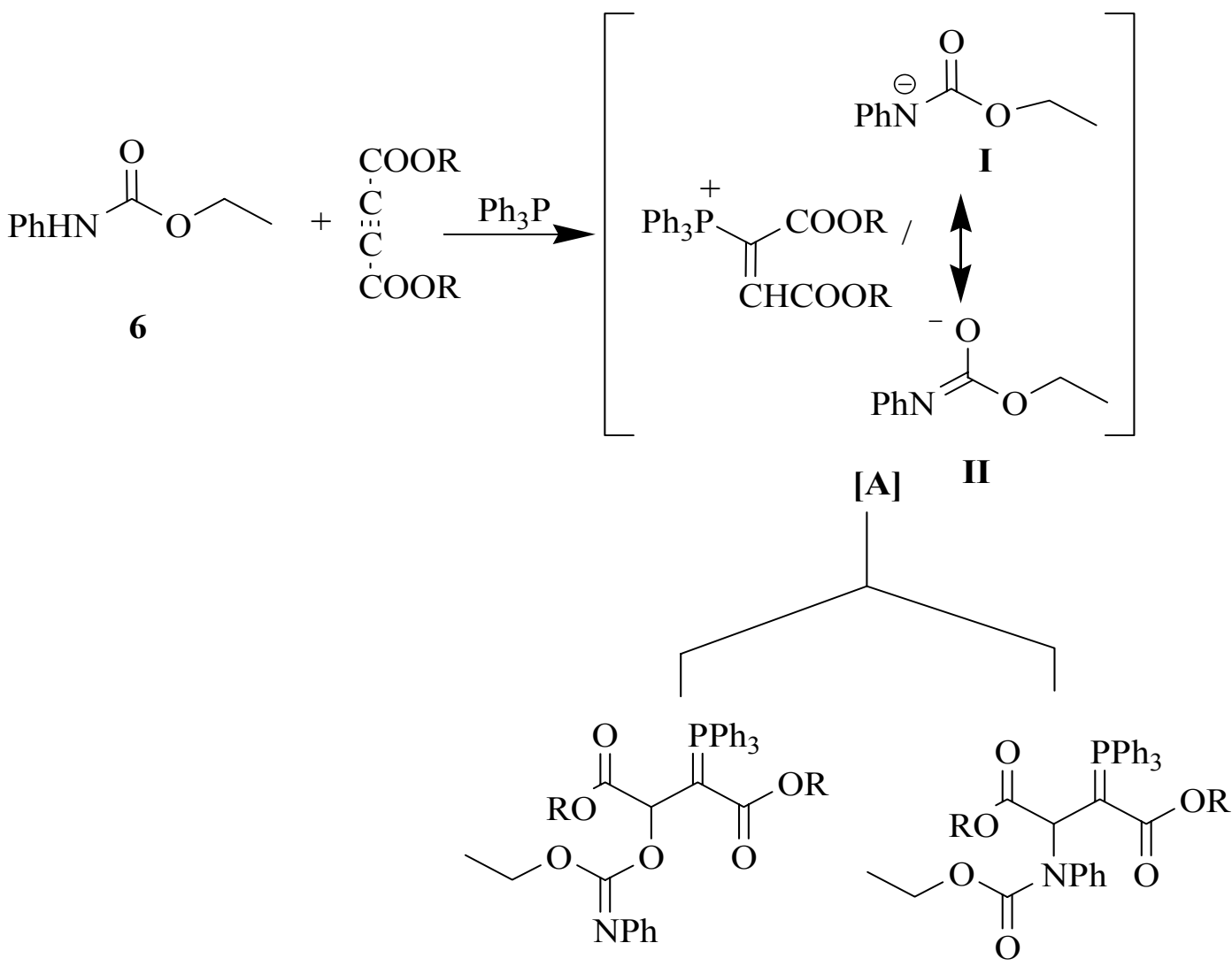

8

not formed 7a $\mathrm{R}=\mathrm{Me}$

$7 \mathbf{b} \quad \mathrm{R}=\mathrm{Et}$

\section{Scheme 2}

The reaction of ethyl phenylcarbamate $\mathbf{6}$ with dialkyl acetylenedicarboxylate takes place with formation of an intermediate, A. As shown in Scheme 2, combination of the ambident anion with phosphonium ion should provide compounds $\mathbf{7}$ and $\mathbf{8}$. We expected $\mathbf{8}$ to be formed as the major product due to its high electron density at the oxygen, but 7 was formed as the only product. The two different reaction pathways are dependent on the strength or weakness of the nucleophile, as well as its softness or hardness as a base, as defined by the "hard and soft acids and bases (HSAB) principle'. ${ }^{27,28}$ Under the present conditions the compound 7 is formed in a chemospecific manner.

Compounds 5a-b and 7a-b were characterized on the basis of their ${ }^{1} \mathrm{H}-$ and ${ }^{13} \mathrm{C}$ NMR, IR, and elemental analysis data, which are consistent with the presence of two rotational isomers. The ylide moiety in these compounds is strongly conjugated with the adjacent carbonyl group, 
and rotation about the partial double bond in the 5-(E), 5-(Z) and 7-(E), 7-(Z) geometrical isomers is slow at room temperature (Scheme 3 ).

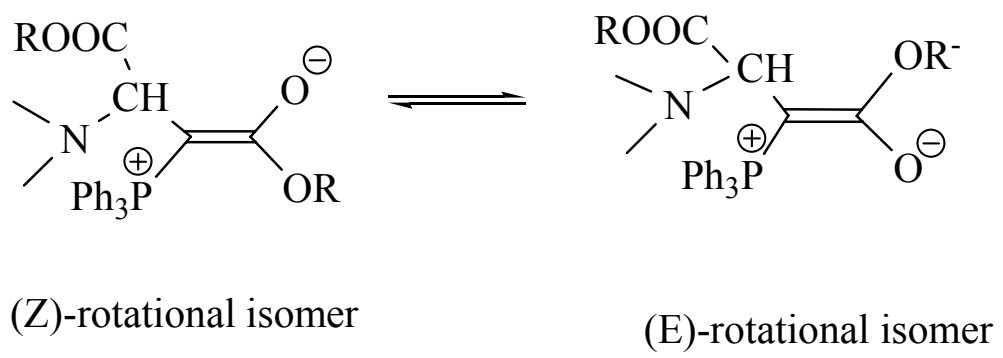

\section{Scheme 3}

The ${ }^{1} \mathrm{H}$ NMR spectrum of compound 5a showed four sharp lines due to the methoxy protons, at $\delta=3.13,3.57,3.68$ and 3.75 , along with signals for methine protons at $\delta=4.50$ and 4.54, which appear as two doublets of doublets $\left({ }^{3} J_{\mathrm{PH}} 15.0 \mathrm{~Hz}\right.$ and $\left.{ }^{3} J_{\mathrm{HH}} 8.7 \mathrm{~Hz}\right)$ and $\left({ }^{3} J_{\mathrm{PH}} 13.3 \mathrm{~Hz}\right.$ and ${ }^{3} J_{\mathrm{HH}} 8.8 \mathrm{~Hz}$ ) respectively for the $Z$ - and $E$ - geometrical isomers. The aromatic protons appear as a multiplet at $\delta=7.47-7.70$ along with fairly broad doublet peak at $\delta=8.03$ for the NH groups. The ${ }^{13} \mathrm{C}$ - NMR spectrum of 5a displayed signals in agreement with the mixture of two rotational isomers. Although the presence of the ${ }^{31} \mathrm{P}$ nucleus complicates both the ${ }^{1} \mathrm{H}$ - and ${ }^{13} \mathrm{C}$ NMR spectra of 5a, it helps in the assignment of signals by long-range spin-spin couplings with ${ }^{1} \mathrm{H}$ - and ${ }^{13} \mathrm{C}$ nuclei. The ${ }^{1} \mathrm{H}$ - and ${ }^{13} \mathrm{C}$ NMR spectra of compound $\mathbf{5 b}$ are similar to those of $\mathbf{5 a}$, with the obvious differences in the ester groups. The ${ }^{1} \mathrm{H}$ - and ${ }^{13} \mathrm{C}$ NMR spectra of compounds $7 \mathbf{a}-7 \mathbf{b}$ displayed signals in agreement with the proposed structures. For example, the ${ }^{1} \mathrm{H}$ NMR spectrum of compound 7a showed four sharp lines due to the methoxy protons at $\delta=2.88,3.11,3.77$ and 3.86 , along with a signal for methine protons at $\delta=4.94$. The protons of ethyl group appeared as a triplet at $\delta=1.00\left({ }^{3} J_{\mathrm{HH}} 7.1 \mathrm{~Hz}\right)$ and a multiplet at $\delta=3.97-4.01$. The aromatic protons appear as a multiplet at $\delta=7.26-7.59$ (see Experimental Section).

\section{Conclusions}

In conclusion, we have demonstrated that the reaction of triphenylphosphine with acetylenic esters in the presence of a compound containing several functional groups can be performed in a chemospecific manner, and the corresponding phosphorus ylides can be synthesized in good yields under mild conditions. 


\section{Experimental Section}

General Procedures. Dialkyl acetylenedicarboxylates and triphenylphosphine were obtained from Merck Chemical Co. and used without further purification. Compounds $\mathbf{1}$ and $\mathbf{6}$ were prepared according to the literature. ${ }^{29}$ Melting points were obtained on a Gallenkamp melting point apparatus and are uncorrected. Elemental analyses for $\mathrm{C}, \mathrm{H}$ and $\mathrm{N}$ were performed at the University of Tehran using a Heraeus CHN-O-Rapid analyzer. IR spectra were measured on a Mattson 1000 FT-IR spectrometer. ${ }^{1} \mathrm{H}$ - and ${ }^{13} \mathrm{C}$ NMR spectra were recorded on a BRUKER DRX-500 AVANCE spectrometer at 500 and $125.77 \mathrm{MHz}$, respectively. Throughout this section, an asterisk $(*)$ denotes the presence of two rotamers

Dimethyl 2-[(2-bromoacetyl)amino]-3-(1,1,1-triphenyl- $\lambda^{5}$-phosphanylidene) succinate (5a). At ambient temperature, dimethyl acetylenedicarboxylate $(0.24 \mathrm{~mL}, 2 \mathrm{mmol})$ was added dropwise to a stirred solution of triphenylphosphine $(0.53 \mathrm{~g}, 2 \mathrm{mmol})$ and 2-bromoacetamide $(0.27 \mathrm{~g}, 2 \mathrm{mmol})$ in a mixture of hexane-ethyl acetate $(6 \mathrm{~mL}, 1: 2)$. After the addition was complete (approximately 5 minutes) the mixture was stirred for an additional $1 \mathrm{~h}$ and was subsequently filtered. The solid collected in the filter was washed thoroughly with ethyl acetate to give a white powder. $\left(0.81 \mathrm{~g}, \mathrm{mp} 170-172{ }^{\circ} \mathrm{C}\right.$, yield $\left.75 \%\right)$; IR $(\mathrm{KBr})\left(v_{\max }, \mathrm{cm}^{-1}\right): 3305(\mathrm{NH})$, 1741 and $1666(\mathrm{C}=\mathrm{O})$. Major isomer, $(\boldsymbol{Z})-5 \mathrm{a},(67 \%),{ }^{1} \mathrm{H}$ NMR: $\delta 3.13$ and $3.68(6 \mathrm{H}, 2 \mathrm{~s}, 2 \mathrm{x}$ $\left.\mathrm{OCH}_{3}\right), 3.72-3.84\left(4 \mathrm{H}, \mathrm{m}, 2 \mathrm{x} \mathrm{CH} \mathrm{CBr}^{*}, 4.50\left(1 \mathrm{H}, \mathrm{dd},{ }^{3} J_{\mathrm{PH}} 15.0 \mathrm{~Hz},{ }^{3} J_{\mathrm{HH}} 8.7 \mathrm{~Hz}, \mathrm{P}=\mathrm{C}-\mathrm{CH}\right), 7.47-\right.$ $7.70(30 \mathrm{H}, \mathrm{m}$, arom. $) *, 8.03\left(2 \mathrm{H}, \mathrm{d},{ }^{2} J_{\mathrm{HH}} 8.8 \mathrm{~Hz}, 2 \mathrm{NH}\right) *{ }^{13} \mathrm{C} \mathrm{NMR}: \delta 29.09\left(\mathrm{CH}_{2} \mathrm{Br}\right), 42.86(\mathrm{~d}$, $\left.{ }^{1} J_{\mathrm{PC}} 129.1 \mathrm{~Hz}, \mathrm{P}=\mathrm{C}\right), 49.11$ and $52.34\left(2 \mathrm{xOCH}_{3}\right), 52.58\left(\mathrm{~d},{ }^{2} J_{\mathrm{PC}} 7.17 \mathrm{~Hz}, \mathrm{P}=\mathrm{C}-\mathrm{CH}\right), 126.35(\mathrm{~d}$, $\left.{ }^{1} J_{\mathrm{pc}} 94.5 \mathrm{~Hz}, \mathrm{C}_{\text {ipso }}\right), 128.68\left(\mathrm{~d},{ }^{3} J_{\mathrm{pc}} 12.3 \mathrm{~Hz}, \mathrm{C}_{\text {meta }}\right)^{*}, 132.16\left(\mathrm{~d},{ }^{4} J_{\mathrm{pc}} 2.4 \mathrm{~Hz}, \mathrm{C}_{\text {para }}\right)^{*}, 133.77(\mathrm{~d}$, $\left.{ }^{2} J_{\mathrm{pc}} 10.0 \mathrm{~Hz}, \mathrm{C}_{\text {ortho }}\right) * 164.41(\mathrm{C}=\mathrm{O}), 170.31\left(\mathrm{~d},{ }^{2} J_{\mathrm{PC}} 12.7 \mathrm{~Hz}, \mathrm{C}=\mathrm{O}\right), 173.13\left(\mathrm{~d},{ }^{3} J_{\mathrm{PC}} 5.7 \mathrm{~Hz}, 2\right.$ $\mathrm{C}=\mathrm{O}) *$. Minor isomer, $(\boldsymbol{E})-5 \mathrm{a}(33 \%),{ }^{1} \mathrm{H}$ NMR: $\delta 3.57$ and $3.75\left(6 \mathrm{H}, 2 \mathrm{~s}, 2 \mathrm{OCH}_{3}\right), 4.54(1 \mathrm{H}$, dd, $\left.{ }^{3} J_{\mathrm{PH}} 13.3 \mathrm{~Hz},{ }^{3} J_{\mathrm{HH}} 8.8 \mathrm{~Hz}, \mathrm{P}=\mathrm{C}-\mathrm{CH}\right) .{ }^{13} \mathrm{C}$ NMR: $\delta 29.20\left(\mathrm{CH}_{2} \mathrm{Br}\right), 43.52\left(\mathrm{~d},{ }^{1} J_{\mathrm{PC}} 136.9 \mathrm{~Hz}, \mathrm{P}=\mathrm{C}\right.$ ), 50.09 and $52.33\left(2 \mathrm{x} \mathrm{OCH}_{3}\right), 51.84\left(\mathrm{~d},{ }^{2} J_{\mathrm{PC}} 17.0 \mathrm{~Hz}, \mathrm{P}=\mathrm{C}-\mathrm{CH}\right), 125.90\left(\mathrm{~d},{ }^{1} J_{\mathrm{pc}} 93.3 \mathrm{~Hz}, \mathrm{C}_{i p s o}\right)$, $163.92(\mathrm{C}=\mathrm{O}), 170.07\left(\mathrm{~d},{ }^{2} J_{\mathrm{PC}} 16.8 \mathrm{~Hz}, \mathrm{C}=\mathrm{O}\right)$. Anal. Calcd. For $\mathrm{C}_{26} \mathrm{H}_{25} \mathrm{BrNO}_{5} \mathrm{P}$ (542.37): $\mathrm{C}$, $57.58 ; \mathrm{H}, 4.65 ; \mathrm{N}, 2.58 \%$. Found: C, 57.46; H, 4.55; N, 2.66\%.

Diethyl 2-[(2-bromo acetyl)amino]-3-(1,1,1-triphenyl- $\lambda^{5}$-phosphanylidene) succinate (5b). Obtained as a white powder from the reaction of diethyl acetylenedicarboxylate $(0.24 \mathrm{~mL}$, $2 \mathrm{mmol})$ with triphenylphosphine $(0.53 \mathrm{~g}, 2 \mathrm{mmol})$ and 2-bromoacetamide $(0.27 \mathrm{~g}, 2 \mathrm{mmol})$ in a mixture of hexane-ethyl acetate $(6 \mathrm{~mL}, 1: 2) .\left(0.79 \mathrm{~g}, \mathrm{mp} 162-164{ }^{\circ} \mathrm{C}\right.$, yield $\left.70 \%\right)$; IR $(\mathrm{KBr})$ $\left(v_{\max }, \mathrm{cm}^{-1}\right): 3310(\mathrm{NH}), 1743$ and $1668(\mathrm{C}=\mathrm{O})$. Major isomer, $(\boldsymbol{Z})-\mathbf{5 b}(64 \%),{ }^{1} \mathrm{H}$ NMR $\delta 0.45$ $\left(3 \mathrm{H}, \mathrm{t},{ }^{3} J_{\mathrm{HH}} 7.0 \mathrm{~Hz}, \mathrm{CH}_{3}\right), 1.22\left(3 \mathrm{H}, \mathrm{t},{ }^{3} \mathrm{~J}_{\mathrm{HH}} 7.1 \mathrm{~Hz}, \mathrm{CH}_{3}\right), 3.68-3.86\left(4 \mathrm{H}, \mathrm{m}, 2 \mathrm{x} \mathrm{CH}_{2} \mathrm{Br}\right)$, * 4.02$4.21\left(8 \mathrm{H}, \mathrm{m}, 4 \mathrm{x} \mathrm{OCH}_{2}\right), * 4.46\left(1 \mathrm{H}, \mathrm{dd},{ }^{3} J_{\mathrm{PH}} 15.0 \mathrm{~Hz},{ }^{3} J_{\mathrm{HH}} 8.5 \mathrm{~Hz}, \mathrm{P}=\mathrm{C}-\mathrm{CH}\right), 7.46-7.75(30 \mathrm{xH}$, $\mathrm{m}$, arom) $* 8.06\left(2 \mathrm{xH}, \mathrm{d},{ }^{3} \mathrm{JHH}_{\mathrm{HH}} 8.5 \mathrm{~Hz}, \mathrm{NH}\right) * .{ }^{13} \mathrm{C}$ NMR: $\delta 13.92$ and $14.18\left(2 \mathrm{x} \mathrm{CH}_{3}\right), 29.11$ $\left(\mathrm{CH}_{2} \mathrm{Br}\right), 42.70\left(\mathrm{~d},{ }^{1} J_{\mathrm{PC}} 129.1 \mathrm{~Hz}, \mathrm{P}=\mathrm{C}\right), 52.54\left(\mathrm{~d},{ }^{2} J_{\mathrm{PC}} 18.8 \mathrm{~Hz}, \mathrm{P}=\mathrm{C}-\mathrm{CH}\right), 57.65$ and $61.17(2 \mathrm{x}$ $\left.\mathrm{OCH}_{2}\right), 126.71\left(\mathrm{~d},{ }^{1} J_{\mathrm{PC}} 92.4 \mathrm{~Hz}, \mathrm{C}_{i p s o}\right), 128.56\left(\mathrm{~d},{ }^{3} J_{\mathrm{PC}} 12.6 \mathrm{~Hz}, \mathrm{C}_{\text {meta }}\right), 132.08\left(\mathrm{~d},{ }^{4} J_{\mathrm{PC}} 2.6 \mathrm{~Hz}\right.$, $\left.\mathrm{C}_{\text {para }}\right)^{*}, 133.87\left(\mathrm{~d},{ }^{2} J_{\mathrm{PC}} 10.0 \mathrm{~Hz}, \mathrm{C}_{\text {ortho }}\right)^{*}, 164.42(\mathrm{C}=\mathrm{O}), 169.87\left(\mathrm{~d},{ }^{2} J_{\mathrm{PC}} 12.6 \mathrm{~Hz}, \mathrm{C}=\mathrm{O}\right), 172.57$ 
$\left(\mathrm{d},{ }^{3} J_{\mathrm{PC}} 7.9 \mathrm{~Hz}, 2 \mathrm{x} \mathrm{C}=\mathrm{O}\right) *$. Minor isomer, $(\boldsymbol{E}) \mathbf{- 5 b}(36 \%),{ }^{1} \mathrm{H}$ NMR: $\delta 1.23\left(3 \mathrm{x} \mathrm{H}, \mathrm{t},{ }^{3} J_{\mathrm{HH}} 7.1\right.$ $\left.\mathrm{Hz}, \mathrm{CH}_{3}\right), 1.25\left(3 \mathrm{x} \mathrm{H}, \mathrm{t},{ }^{3} J_{\mathrm{HH}} 7.1 \mathrm{~Hz}, \mathrm{CH}_{3}\right), 4.47\left(1 \mathrm{H}, \mathrm{dd},{ }^{3} J_{\mathrm{PH}} 15.5 \mathrm{~Hz},{ }^{3} J_{\mathrm{HH}} 6.5 \mathrm{~Hz}, \mathrm{P}=\mathrm{C}-\mathrm{CH}\right)$. ${ }^{13} \mathrm{C}$ NMR: $\delta 14.19$ and $15.05(2 \mathrm{x} \mathrm{CH}), 29.29\left(\mathrm{CH}_{2} \mathrm{Br}\right), 43.64\left(\mathrm{~d},{ }^{1} J_{\mathrm{PC}} 137.1 \mathrm{~Hz}, \mathrm{P}=\mathrm{C}\right), 51.90(\mathrm{~d}$, $\left.{ }^{2} J_{\mathrm{PC}} 17.6 \mathrm{~Hz}, \mathrm{P}=\mathrm{C}-\mathrm{CH}\right), 58.25$ and $61.18\left(2 \mathrm{x} \mathrm{OCH}_{2}\right), 126.06\left(\mathrm{~d},{ }^{1} J_{\mathrm{pc}} 91.8 \mathrm{~Hz}, \mathrm{C}_{i p s o}\right), 128.65(\mathrm{~d}$, $\left.{ }^{3} J_{\mathrm{pc}} 13.1 \mathrm{~Hz}, \mathrm{C}_{\text {meta }}\right), 163.77(\mathrm{C}=\mathrm{O}), 169.82\left(\mathrm{~d},{ }^{2} J_{\mathrm{PC}} 20.0 \mathrm{~Hz}, \mathrm{C}=\mathrm{O}\right)$. Anal. Calcd. For $\mathrm{C}_{28} \mathrm{H}_{29} \mathrm{BrNO}_{5} \mathrm{P}$ (570.42): C, 58.96; H, 5.12; N, 2.46. Found: C, 59.06; H, 5.05; N, 2.50\%.

Dimethyl 2-[N-ethoxycarbonyl- $N$-phenylamino]-3-(1,1,1-triphenyl- $\lambda^{5}$-phosphanylidene)succinate (7a). Obtained as a white powder from the reaction of dimethyl acetylenedicarboxylate $(0.24 \mathrm{~mL}, 2 \mathrm{mmol})$ with triphenylphosphine $(0.53 \mathrm{~g}, 2 \mathrm{mmol})$ and ethyl phenylcarbamate $(0.33 \mathrm{~g}, 2 \mathrm{mmol})$ in a mixture of hexane-ethyl acetate $(6 \mathrm{~mL}, 1: 2) .0 .61 \mathrm{~g}, \mathrm{~m} . \mathrm{p}$. 161-164 ${ }^{\circ} \mathrm{C}$, yield 75\%); IR (KBr) $\left(v_{\max }, \mathrm{cm}^{-1}\right): 1741$ and $1691(\mathrm{C}=\mathrm{O}) .(Z)-7 \mathbf{a}(50 \%),{ }^{1} \mathrm{H}$ NMR: $\delta$ $1.00\left(6 \mathrm{H}, \mathrm{t},{ }^{3} J_{\mathrm{HH}} 7.1 \mathrm{~Hz}, \mathrm{CH}_{3}\right)^{*}, 2.88$ and $3.77\left(6 \mathrm{H}, 2 \mathrm{~s}, 2 \mathrm{x} \mathrm{OCH}_{3}\right), 3.97-4.01(4 \mathrm{H}, \mathrm{m}, 2 \mathrm{x}$ $\left.\mathrm{OCH}_{2}\right)^{*}, 4.94(\mathrm{P}=\mathrm{C}-\mathrm{CH})^{*}, 7.26-7.59(40 \mathrm{H}, \mathrm{m} \text {, arom })^{*} .{ }^{13} \mathrm{C}^{13} \mathrm{NR}: \delta 14.53\left(2 \mathrm{x} \mathrm{CH}_{3}\right)^{*}, 41.28(\mathrm{~d}$, $\left.{ }^{1} J_{\mathrm{PC}} 135.1 \mathrm{~Hz}, 2 \mathrm{x} \mathrm{P}=\mathrm{C}\right) *, 48.76$ and $52.04\left(2 \mathrm{x} \mathrm{OCH}_{3}\right), 60.93\left(\mathrm{OCH}_{2}\right), 61.05\left(\mathrm{~d},{ }^{2} J_{\mathrm{PC}} 16.1 \mathrm{~Hz}\right.$, $\mathrm{P}=\mathrm{C}-\mathrm{CH}), 126.35\left(\mathrm{~d},{ }^{1} J_{\mathrm{PC}} 94.5 \mathrm{~Hz}, \mathrm{C}_{\text {ipso }}\right), 126.53,127.31$ and $129.69(3 \mathrm{x} \mathrm{CH}), 128.58\left(\mathrm{~d},{ }^{3} J_{\mathrm{PC}}\right.$ $\left.12.3 \mathrm{~Hz}, \mathrm{C}_{\text {meta }}\right), 131.94$ (d, $\left.{ }^{4} J_{\mathrm{PC}} 2.5 \mathrm{~Hz}, \mathrm{C}_{\text {para }}\right), 133.56$ (d, $\left.{ }^{2} J_{\mathrm{PC}} 9.7 \mathrm{~Hz}, \mathrm{C}_{\text {ortho }}\right), 139.21$ (C), 154.98 $(\mathrm{C}=\mathrm{O}), 168.50\left(\mathrm{~d},{ }^{2} J_{\mathrm{PC}} 13.8 \mathrm{~Hz}, \mathrm{C}=\mathrm{O}\right), 173.62(\mathrm{C}=\mathrm{O}) * .(\boldsymbol{E})-7 \mathbf{a}(50 \%),{ }^{1} \mathrm{H}$ NMR: $\delta 3.11$ and 3.86 $(6 \mathrm{H}, 2 \mathrm{~s}, 2 \mathrm{x} \mathrm{OCH}),{ }^{13} \mathrm{C}$ NMR: $\delta 49.41$ and $52.33\left(2 \mathrm{x} \mathrm{OCH}_{3}\right), 61.02\left(\mathrm{OCH}_{2}\right), 61.86\left(\mathrm{~d},{ }^{2} J_{\mathrm{PC}} 16.4\right.$ $\mathrm{Hz}, \mathrm{P}=\mathrm{C}-\mathrm{CH}), 126.56\left(\mathrm{~d},{ }^{1} J_{\mathrm{PC}} 93.1 \mathrm{~Hz}, \mathrm{C}_{i p s o}\right), 127.32,127.62$ and $131.18(3 \mathrm{x} \mathrm{CH}), 128.68(\mathrm{~d}$, $\left.{ }^{3} J_{\mathrm{PC}} 12.6 \mathrm{~Hz}, \mathrm{C}_{\text {meta }}\right), 132.04$ (d, $\left.{ }^{4} J_{\mathrm{PC}} 2.5 \mathrm{~Hz}, \mathrm{C}_{\mathrm{para}}\right), 133.77$ (d, $\left.{ }^{2} J_{\mathrm{PC}} 9.8 \mathrm{~Hz}, \mathrm{C}_{\text {ortho }}\right), 139.46$ (C), 155.34 (C=O), $170.04\left(\mathrm{~d},{ }^{2} J_{\mathrm{PC}} 18.9 \mathrm{~Hz}, \mathrm{C}=\mathrm{O}\right)$. Anal. Calcd. for $\mathrm{C}_{33} \mathrm{H}_{32} \mathrm{NO}_{6} \mathrm{P}(565)$ : C, 69.59; $\mathrm{H}$, $5.66 ; \mathrm{N}, 2.46 \%$. Found: C, 69.49; H, 5.62; N, 2.51\%.

Diethyl 2-[N-ethoxycarbonyl- $N$-phenylamino]-3-(1,1,1-triphenyl- $\lambda^{5}$-phosphanylidene)succinate (7b). A white powder from the reaction of diethyl acetylenedicarboxylate $(0.24 \mathrm{~mL}$, $2 \mathrm{mmol})$ with triphenylphosphine $(0.53 \mathrm{~g}, 2 \mathrm{mmol})$ and ethyl phenylcarbamate $(0.33 \mathrm{~g}, 2 \mathrm{mmol})$ in a mixture of hexane-ethyl acetate $(6 \mathrm{~mL}, 1: 2) .\left(0.61 \mathrm{~g}\right.$, m.p. $166-170{ }^{\circ} \mathrm{C}$, yield $\left.71 \%\right)$; IR $(\mathrm{KBr})$ $\left(v_{\max }, \mathrm{cm}^{-1}\right): 1751$ and $1681(\mathrm{C}=\mathrm{O})$. Major isomer, $(\boldsymbol{Z})-7 \mathbf{b}(53 \%),{ }^{1} \mathrm{H}$ NMR: $\delta 0.31\left(3 \mathrm{H}, \mathrm{t},{ }^{3} J_{\mathrm{HH}}\right.$ $\left.7.1 \mathrm{~Hz}, 2 \mathrm{x} \mathrm{CH}_{3}\right), 0.97\left(6 \mathrm{H}, \mathrm{t},{ }^{3} J_{\mathrm{HH}} 7.0 \mathrm{~Hz}, 2 \mathrm{x} \mathrm{CH}_{3}\right), 1.33\left(3 \mathrm{H}, \mathrm{t},{ }^{3} J_{\mathrm{HH}} 8.3 \mathrm{~Hz}\right), 3.34-4.44(12 \mathrm{H}$, $\left.\mathrm{m}, 6 \mathrm{x} \mathrm{OCH}_{2}\right), 4.93(\mathrm{P}=\mathrm{C}-\mathrm{CH})^{*}, 7.25-7.61(40 \mathrm{H}, \mathrm{m} \text {, arom })^{*} .{ }^{13} \mathrm{C}$ NMR: $\delta 13.89$ and $14.38(2 \mathrm{x}$ $\left.\mathrm{CH}_{3}\right), 14.55\left(2 \mathrm{CH}_{3}\right)^{*}, 41.65\left(\mathrm{~d},{ }^{1} J_{\mathrm{PC}} 134.7 \mathrm{~Hz}, \mathrm{P}=\mathrm{C}\right), 57.37$ and $60.77\left(2 \mathrm{x} \mathrm{OCH}_{2}\right), 60.86$ $\left(\mathrm{OCH}_{2}\right)^{*}, 60.98\left(\mathrm{~d},{ }^{2} J_{\mathrm{PC}} 17.5 \mathrm{~Hz}, \mathrm{P}=\mathrm{C}-\mathrm{CH}\right), 126.33\left(\mathrm{~d},{ }^{1} J_{\mathrm{PC}} 94.7 \mathrm{~Hz}, \mathrm{C}_{i p s o}\right), 126.71,127.71$ and $129.95(3 \mathrm{x} \mathrm{CH}), 128.48$ (d, $\left.{ }^{3} J_{\mathrm{PC}} 12.2 \mathrm{~Hz}, \mathrm{C}_{\text {meta }}\right), 131.87$ (d, $\left.{ }^{4} J_{\mathrm{PC}} 2.5 \mathrm{~Hz}, \mathrm{C}_{\mathrm{para}}\right), 133.68$ (d, ${ }^{2} J_{\mathrm{PC}} 9.8$ $\left.\mathrm{Hz}, \mathrm{C}_{\text {ortho }}\right), 139.29(\mathrm{C}), 155.38(\mathrm{C}=\mathrm{O}), 167.95\left(\mathrm{~d},{ }^{2} J_{\mathrm{PC}} 13.0 \mathrm{~Hz}, \mathrm{C}=\mathrm{O}\right), 172.77(\mathrm{C}=\mathrm{O})^{*}$. Minor isomer, $(\boldsymbol{E})-7 \mathbf{b} \quad(47 \%),{ }^{1} \mathrm{H}$ NMR: $\delta 0.99\left(3 \mathrm{H}, \mathrm{t},{ }^{3} J_{\mathrm{HH}} 7.1 \mathrm{~Hz}, \mathrm{CH}_{3}\right), 1.36\left(3 \mathrm{H}, \mathrm{t},{ }^{3} J_{\mathrm{HH}} 7.3 \mathrm{~Hz}\right.$, $\left.\mathrm{CH}_{3}\right) .{ }^{13} \mathrm{C}$ NMR: $\delta 14.30$ and $14.45\left(2 \mathrm{OCH}_{3}\right), 38.98\left(\mathrm{~d},{ }^{1} \mathrm{~J}_{\mathrm{PC}} 133.4 \mathrm{~Hz}, \mathrm{P}=\mathrm{C}\right), 57.91$ and 60.87 $(2 \mathrm{x} \mathrm{OCH}), 61.91\left(\mathrm{~d},{ }^{3} J_{\mathrm{PC}} 16.7 \mathrm{~Hz}, \mathrm{P}=\mathrm{C}-\mathrm{CH}\right), 126.88\left(\mathrm{~d},{ }^{1} J_{\mathrm{PC}} 91.9 \mathrm{~Hz}, \mathrm{C}_{i p s o}\right), 127.32,127.50$ and $131.37(3 \mathrm{x} \mathrm{CH}), 128.60\left(\mathrm{~d},{ }^{3} J_{\mathrm{PC}} 12.3 \mathrm{~Hz}, \mathrm{C}_{\text {meta }}\right), 131.95\left(\mathrm{~d},{ }^{4} J_{\mathrm{PC}} 2.8 \mathrm{~Hz}, \mathrm{C}_{\text {para }}\right), 133.80(\mathrm{~d}$, $\left.{ }^{2} J_{\mathrm{PC}} 9.8 \mathrm{~Hz}, \mathrm{C}_{\text {ortho }}\right), 139.29$ (C), $155.15(\mathrm{C}=\mathrm{O}), 169.87\left(\mathrm{~d},{ }^{2} J_{\mathrm{PC}} 18.1 \mathrm{~Hz}, \mathrm{C}=\mathrm{O}\right)$. Anal. Calcd. For $\mathrm{C}_{35} \mathrm{H}_{36} \mathrm{NO}_{6} \mathrm{P}$ (597.65): C, 70.34; H, 6.07; N, 2.34. Found: C, 70.40; H, 6.07; N, 2.39\%. 


\section{Acknowledgements}

The authors express appreciation to the Shahid Bahonar University of Kerman Faculty Research committee funds for its support of this investigation.

\section{References}

1. Grayson, M.; Griffith, E. J. Topics in Phosphorus Chemistry, Interscience: New York, 1972; Vol. 7.

2. Maryano, B. E.; Reitz, A. B. Chem. Rev. 1989, 89, 863.

3. Bestmann, H. J.; Vostrowsky, O. Topics Curr. Chem. 1983, 109, 86.

4. Schobert, R.; Siegfried, S.; Nieuwenhuyzen, M.; Milius, W.; Hampel, F. J. Chem. Soc., Perkin Trans. 1 2000, 1723.

5. Yavari, I.; Islami, M. R. J. Chem. Res. (S) 1998, 166.

6. Balema, V. P.; Wiench, J. W.; Pruski, M.; Pecharsky, V. K. J. Am. Chem. Soc. 2002, 124, 6244.

7. Balema, V. P.; Wiench, J. W.; Pruski, M.; Pecharsky, V. K. Chem. Commun. 2002, 7, 724.

8. Yavari, I.; Islami, M. R. Phosphorus Sulfur Silicon Relat. Elem. 1997, 130, 229.

9. Maryanoff, B. E.; Reitz, A. B. Chem. Rev. 1989, 89, 863.

10. Russell, M. G.; Warren, S. J. Chem. Soc. PerkinTrans 1 2000, 505.

11. Greenwald, R.; Chaykovsky, M.; Corey, E. J. J. Org. Chem. 1963, 28, 1128.

12. Fitjer, L.; Quabeck, U. Synth. Commun. 1985, 15, 855.

13. Yavari, I.; Samzadeh-Kermani, A. R. Tetrahedron Lett. 1998, 39, 6343.

14. Ramazani, A.; Souldozi, A. Phosphorus Sulfur Silicon Relat. Elem. 2005, 180, 2801.

15. Yavari, I.; Samzadeh-Kermani, A. R. Tetrahedron Lett. 1998, 39, 6343.

16. Yavari, I.; Alizadeh, A. A. Monatsh. Chem. 2003, 134, 435.

17. Yavari, I.; Islami, M. R.; Bijanzadeh, H. R. Terahedron 1999, 55, 5547.

18. Kalantari, M.; Islami, M. R.; Hassani, Z.; Saidi, K. ARKIVOC 2006, (x), 55.

19. Islami, M. R.; Abedini-Torghabeh, J.; Fatemi, S. J.; Hassani, Z.; Amiry, A. Synlett 2004, 1707.

20. Islami M. R.; Hassani, Z.; Sheibani, H.; Abdolahzadeh, B.; Etminan, N. Tetrahedron 2003, 59, 4993.

21. Wittig, G.; Schoellkopf, U. Org. Synth. 1973, (V), 751.

22. Kreutzkamp, N.; Kayser, H. Chem. Ber. 1956, 9, 1614.

23. Borowitz, I. J.; Parnes, H. J. Org. Chem. 1967, 32, 3560.

24. Downie, I. M.; Holmes, J. B.; Lee, J.B. Chem. and Ind. 1966, 900.

25. Magid, R. M.; Fruchey, O. S.; Johnson, W. L.; Allen, T. G. J. Org. Chem. 1979, 44, 359

26. Slagle, T. D.; Huang, T. T. S.; Franzus, B. J. Org. Chem. 1981, 46, 3526. 
27. Ho, T. L. Hard and Soft Acids and Bases Principles in Organic Chemistry, Academic Press: New York, 1977.

28. Pearson, R. G. Songstad, J. J. Am. Chem. Soc. 1967, 89, 1827.

29. Mizuno, M.; Yamano, M. Org. Synth. 2007, 84, 325. 